\title{
SECOND ORDER ERGODIC THEOREMS FOR ERGODIC TRANSFORMATIONS OF INFINITE MEASURE SPACES
}

\author{
JON AARONSON, MANFRED DENKER, AND ALBERT M. FISHER
}

(Communicated by Andrew M. Bruckner)

Abstract. For certain pointwise dual ergodic transformations $T$ we prove almost sure convergence of the log-averages

$$
\frac{1}{\log N} \sum_{n=1}^{N} \frac{1}{n a(n)} \sum_{k=1}^{n} f \circ T^{k} \quad\left(f \in L_{1}\right)
$$

and the Chung-Erdös averages

$$
\frac{1}{\log a(N)} \sum_{k=1}^{N} \frac{1}{a(k)} f \circ T^{k} \quad\left(f \in L_{1}^{+}\right)
$$

towards $\int f$, where $a(n)$ denotes the return sequence of $T$.

\section{INTRODUCTION}

Let $(\Omega, \mathscr{F}, m, T)$ be a conservative, ergodic infinite measure preserving dynamical system, where $m$ is a nonatomic, $\sigma$-finite, infinite measure.

For a measurable function $f$ denote

$$
S_{n} f=\sum_{k=1}^{n} f \circ T^{k}
$$

We are interested in the behaviour of sums of the form

$$
\frac{1}{\log N} \sum_{n=1}^{N} \frac{S_{n} f}{n a(n)}=\frac{1}{\log N} \sum_{n=1}^{N} \frac{1}{n a(n)} \sum_{k=1}^{n} f \circ T^{k}
$$

for some (and hence all) functions $f \in L_{1}(m)$ and

$$
\frac{1}{\log a(n)} \sum_{k=1}^{n} \frac{1}{a(k)} f \circ T^{k}
$$

for functions $f \in L_{1}^{+}(m)$, where $a(n)>0$ are constants. The first sums will be called the log-averages (of the normalized partial sums) and the second sums the Chung-Erdös averages (as they were first studied in [5]).

Received by the editors June 11, 1990.

1980 Mathematics Subject Classification (1985 Revision). Primary 28D05; Secondary 60F15. 
In particular, we are interested in situations where these averages converge to $\int f$ for every $f \in L_{1}^{+}(m)$, for some sequence of normalizing constants $a(n)$. Not every conservative, ergodic measure preserving transformation has this property (see $[2, \S 2 ; 8$, Proposition 2.7]).

Examples where the log-averages converge have been given in $[4,8]$ (compare also [7]). Chung and Erdös [5, Theorem 6] proved that the Chung-Erdös averages converge for any conservative, ergodic Markov shift.

In this paper, we consider the convergence of the averages for pointwise dual ergodic transformations $T[2, \S 1]$. Denote by $\widehat{T}$ the dual operator of $T$ : $L_{\infty}(m) \rightarrow L_{\infty}(m)$, restricted to $L_{1}(m)$. The assumption that $T$ is pointwise dual ergodic means that there are normalizing constants $a(n)$ such that

$$
\lim _{n \rightarrow \infty} \frac{1}{a(n)} \sum_{k=1}^{n} \widehat{T}^{k} f=\int_{\Omega} f d m \quad \text { a.s. }
$$

for every function $f \in L_{1}(m)$. The constants $a(n)$ are given by

$$
a(n) \sim \sum_{k=1}^{n} \int_{A} \widehat{T}^{k} 1_{A} d m,
$$

where $A \in \mathscr{F}$ is a set of measure one such that the convergence in (3) is a.s. and in $L_{1}\left(m_{\mid A}\right)$. The sequence $a(n)$ is called a return sequence for $T$.

Although convergence of the log-averages is not in general equivalent to convergence of the Chung-Erdös averages, we have

Proposition 1. Let $a(n)$ be regularly varying with index $\alpha>0$. Then, for every positive function $f \in L_{1}^{+}(m)$, we have

$$
\lim _{N \rightarrow \infty} \frac{1}{\log a(N)} \sum_{k=1}^{N} \frac{1}{a(k)} f \circ T^{k}=\int f d m \quad \text { a.s. }
$$

if and only if

$$
\lim _{N \rightarrow \infty} \frac{1}{\log N} \sum_{n=1}^{N} \frac{1}{n a(n)} S_{n} f=\int f d m . \quad \text { a.s. }
$$

In general:

Proposition 2. For any $f \in L_{1}^{+}(m)$ the intrinsic averages

$$
\frac{1}{\log a(N)} \sum_{n=1}^{N} \frac{u_{n}}{a(n)^{2}} S_{n} f
$$

converge a.s., where $u_{n}=a(n+1)-a(n)$, if and only if the Chung-Erdös averages (2) converge.

The proofs of these propositions are elementary and left to the reader.

In $\S 2$ we prove second order ergodic theorems for log-averages (1). The method of proof uses an estimate of the variances of (1) in terms of

$$
\Phi(N, \varepsilon)=\sup \left\{\frac{a(n)}{a\left(n N^{\varepsilon}\right)}: 1 \leq n \leq N^{1-\varepsilon}\right\} \quad(N \geq 1, \varepsilon>0) .
$$

We show in Theorem 1 that (1) converges in measure on sets of finite measure if for any $\varepsilon>0, \Phi(N, \varepsilon) \rightarrow 0$ as $N \rightarrow \infty$. Moreover, imposing logarithmic rates 
in this convergence (specfically, condition $(\star)$ in Theorem 2) and a logarithmic asymptotic error in (3) for suitable $f$ 's, we obtain a.s. convergence for the log-averages (1) in Theorem 2.

The conditions on $\Phi(N, \varepsilon)$ are always satisfied if the return sequence $(a(n))_{n \geq 1}$ is regularly varying with positive index. The "asymptotic error" condition always holds for Markov shifts. The theorem of Chung and Erdös states that for Markov chains the averages (2) converge to $\int f d m$ a.s., and hence by Proposition 1, also the log-averages converge a.s., if the return sequence is regularly varying with positive index. In fact, we show in $\S 2$ that a.s. convergence holds for certain slowly varying sequences $a(n)$. This implies that there are Markov shifts for which the log-averages converge a.s., though this cannot be deduced from the convergence of the Chung-Erdös averages by Proposition 1.

As another application of Theorem 2 we obtain that number-theoretical transformations in the sense of Thaler [10] have convergent log-averages when the return sequence $a(n)$ satisfies $(\star)$.

The conclusion of Theorem 2 also applies to the shifts of Markov processes that are recurrent in the sense of Harris, and whose return sequences satisfy $(\star)$. See $[2, \S 1$, Example 2].

The method of proof for the result of Chung and Erdös is a cancellation argument. A similar argument is used in $\S 3$ to show a.s. convergence in (2) for the real restrictions of conservative, Lebesgue measure preserving, odd inner functions of the upper half-plane.

\section{LOG-AVERAGES}

In this section let $T$ denote a pointwise dual ergodic transformation on the nonatomic, $\sigma$-finite, infinite measure space $(\Omega, \mathscr{F}, m)$. Then there exists a set $A \in \mathscr{F}$ of measure one and a sequence $\bar{a}(n)$ such that

$$
a(n) \sim \bar{a}(n) \uparrow \infty
$$

and

$$
\sum_{k=1}^{n} \widehat{T}^{k} 1_{A}(x) \leq \bar{a}(n)
$$

for $x \in A$ and $n \geq 1$. This can be shown by a successive use of Egorov's theorem. Since $a(n)$ may be replaced by an asymptotically equivalent sequence we assume that $a(0)=1$ and

$$
a(n)=1+\int_{A} S_{n} 1_{A} d m .
$$

Write

$$
\frac{\bar{a}(n)}{a(n)}=1+\beta_{n} \quad(n \geq 1) .
$$

We now state our theorems for log-averages.

Theorem 1. Suppose that for every $\varepsilon>0$

$$
\lim _{N \rightarrow \infty} \Phi(N, \varepsilon)=0 .
$$


Then, for any $f \in L_{1}(m)$,

$$
\lim _{N \rightarrow \infty} \frac{1}{\log N} \sum_{n=1}^{N} \frac{1}{n a(n)} \sum_{k=1}^{n} f \circ T^{k}=\int_{\Omega} f d m
$$

in measure on any set of finite measure.

Theorem 2. Suppose that

(*) $\exists \varepsilon, \gamma>0 \quad \ni \quad \Phi\left(N,(\log N)^{-\gamma}\right)=O\left(\frac{1}{(\log N)^{\varepsilon}}\right) \quad$ as $N \rightarrow \infty$.

Moreover, assume that $\beta_{N}$ defined in (10) satisfies

$$
\beta_{N}=O\left(\frac{1}{(\log N)^{\gamma}}\right) \text { as } N \rightarrow \infty \text {. }
$$

Then for every $f \in L_{1}(m)$

$$
\lim _{N \rightarrow \infty} \frac{1}{\log N} \sum_{n=1}^{N} \frac{1}{n a(n)} \sum_{k=1}^{n} f \circ T^{k}=\int_{\Omega} f d m \quad \text { a.s. }
$$

Corollary 1. Let $(\Omega, \mathscr{F}, m, T)$ be a conservative Markov shift with return sequence $a(n)$ satisfying $(\star)$. Then for every function $f \in L_{1}(m)$

$$
\lim _{N \rightarrow \infty} \frac{1}{\log N} \sum_{n=1}^{N} \frac{1}{n a(n)} S_{n} f=\int_{\Omega} f d m \quad \text { a.s. }
$$

and

$$
\lim _{N \rightarrow \infty} \frac{1}{\log a(N)} \sum_{k=1}^{N} \frac{1}{a(k)} f \circ T^{k}=\int_{\Omega} f d m \quad \text { a.s. }
$$

Remark 1. Let

$$
a(n)=\exp \left(\int_{1}^{n} \frac{\eta(t)}{t} d t\right) \quad(n \geq 1) .
$$

If $\eta(t)$ is decreasing then $\Phi(N, \varepsilon) \rightarrow 0$ as $N \rightarrow \infty$ for every $\varepsilon>0$ if and only if

$$
\lim _{t \rightarrow \infty} \eta(t) \log t=\infty
$$

and $(\star)$ holds if and only if

$$
\lim _{t \rightarrow \infty} \eta(t)(\log t)^{1-\delta}=\infty
$$

for some $\delta>0$.

There are Markov shifts with slowly varying return sequences satisfying (12). For these Markov shifts, the log-averages converge a.e. by Corollary 1. On the other hand, it can be seen easily that if $T$ is a Markov shift with return sequence $\log N$, then the log-averages do not converge in measure.

Remark 2. Suppose that $T$ is a number-theoretical transformation in the sense of Thaler [10]. Then (see [3]) $T$ is pointwise dual ergodic, and, indeed, there is a set $A$ of measure one such that for all $p, n \geq 1$

$$
\sum_{k=1}^{n} \widehat{T}^{k} 1_{A} \leq p+\left(1+\varepsilon_{p}\right) a(n)
$$


uniformly on $A$, where $\varepsilon_{p}=O\left(\theta^{\sqrt{p}}\right)$ for some $\theta<1$.

Suppose that this is the case with $\varepsilon_{p}=O\left((\log p)^{-\delta}\right)$ and that $a(n)$ satisfies $(\star)$. Then $\log a(n) \geq(\varepsilon / 2) \log n$ for $n$ large, where $\varepsilon$ is as in $(\star)$.

Set $p_{n}=a(n)(\log n)^{-\varepsilon / 4}$. Then

$$
\begin{aligned}
\sum_{k=1}^{n} \widehat{T}^{k} 1_{A} & \leq\left(1+\frac{p_{n}}{a(n)}+O\left(\left(\log p_{n}\right)^{-\delta}\right)\right) a(n) \\
& =\left(1+(\log n)^{-\varepsilon / 4}+O\left(\left(\log p_{n}\right)^{-\delta}\right)\right) a(n) .
\end{aligned}
$$

Now $\log p_{n} \geq \log a(n)-(\varepsilon / 4) \log n \geq(\varepsilon / 4) \log n$, and so

$$
\sum_{k=1}^{n} \widehat{T}^{k} 1_{A}=\left(1+O\left((\log n)^{-\min (\varepsilon / 4, \delta)}\right)\right) a(n)
$$

uniformly on $A$ and Theorem 2 applies.

The proofs of our results are based on an estimate of the variance of the log-averages. We denote $S_{n}=\sum_{k=1}^{n} 1_{A} \circ T^{k}(n \geq 1), S_{0}=0, T_{N}=$ $\sum_{n=1}^{N}\left(S_{n} / n a(n)\right)$, and $\widehat{T}_{n}=\sum_{k=1}^{n} \widehat{T}^{k} 1_{A}(n \geq 1), \widehat{T}_{0}=0$. Finally, we shall use the expectation sign $E$ to denote the integral with respect to the measure $m$ over the set $A$.

Lemma 1. If $n \leq m$, then

$$
E S_{n} S_{m} \leq \sum_{k=0}^{n}(a(m-k)+a(n-k)) l_{k},
$$

where $l_{k}=\bar{a}(k)-\bar{a}(k-1)$ and where $\bar{a}(-1)=0$.

Proof. Using the duality of $T$ and $\widehat{T}$, we have

$$
\begin{aligned}
E S_{n} S_{m}=\sum_{k=1}^{n} \sum_{l=1}^{m} \int_{A} 1_{A} \circ T^{k} 1_{A} \circ T^{l} d m \\
=\sum_{k=1}^{n} \int_{A}\left(1_{A} S_{m-k}\right) \circ T^{k} d m+\sum_{k=1}^{n-1} \int_{A}\left(1_{A} S_{n-k}\right) \circ T^{k} d m+a(n)-1 \\
=\sum_{k=1}^{n} \int_{A} \widehat{T}^{k} 1_{A} S_{m-k} d m+\sum_{k=1}^{n-1} \int_{A} \widehat{T}^{k} 1_{A} S_{n-k} d m+a(n)-1 \\
=\sum_{k=1}^{n} \int_{A}\left(\widehat{T}_{k}-\widehat{T}_{k-1}\right) S_{m-k} d m+\sum_{k=1}^{n-1} \int_{A}\left(\widehat{T}_{k}-\widehat{T}_{k-1}\right) S_{n-k} d m+a(n)-1 \\
=\sum_{k=1}^{n} \int_{A} \widehat{T}_{k}\left(S_{m-k}-S_{m-k-1}\right) d m+\sum_{k=1}^{n-1} \int_{A} \widehat{T}_{k}\left(S_{n-k}-S_{n-k-1}\right) d m+a(n)-1 \\
\quad+\int_{A} \widehat{T}_{n} S_{m-n-1} d m .
\end{aligned}
$$


By (8) it follows that

$$
\begin{aligned}
E S_{n} S_{m} \leq & \sum_{k=1}^{n} \bar{a}(k)(a(m-k)-a(m-k-1)) \\
& +\sum_{k=1}^{n-1} \bar{a}(k)(a(n-k)-a(n-k-1))+a(n)+\bar{a}(n) a(m-n-1) \\
\leq & \sum_{k=0}^{n}(a(m-k)+a(n-k))(\bar{a}(k)-\bar{a}(k-1)) .
\end{aligned}
$$

Lemma 2. For every $\varepsilon>0, N \geq 2$, and $N \geq n \geq 1$

$$
\sum_{m=n}^{N} \frac{1}{m a(m)} \leq\left(\varepsilon+\Phi(N, \varepsilon)+(\log N)^{-1}\right) \frac{\log N}{a(n)} \text {. }
$$

Proof. Fix $N \geq 2$. For $n \geq N^{1-\varepsilon}$ it follows that

$$
\sum_{m=n}^{N} \frac{1}{m a(m)} \leq \frac{1}{a(n)}\left(1+\log N-\log N^{1-\varepsilon}\right)=\frac{1}{a(n)}\left(\varepsilon+(\log N)^{-1}\right) \log N .
$$

If $n \leq N^{1-\varepsilon}$, then

$$
\begin{aligned}
\sum_{m=n}^{N} \frac{1}{m a(m)} & \leq \frac{1}{a(n)}\left(\Phi(N, \varepsilon) \sum_{m=\left[n N^{\varepsilon}\right]+1}^{N} \frac{1}{m}+\sum_{m=n}^{\left[n N^{\varepsilon}\right]} \frac{1}{m}\right) \\
& =\left[\varepsilon+\Phi(N, \varepsilon)+(\log N)^{-1}\right] \frac{\log N}{a(n)}
\end{aligned}
$$

Lemma 3. For $N \geq 2$

$$
\begin{aligned}
& \sum_{n=1}^{N} \frac{1}{n a(n)} \sum_{m=n}^{N} \frac{1}{m a(m)} \sum_{k=0}^{n} l_{k} a(n-k) \\
& \left.\quad \leq\left(1+\sup _{n \geq 1} \beta_{n}\right)\right)\left(\varepsilon+\Phi(N, \varepsilon)+(\log N)^{-1}\right)(1+\log N) \log N .
\end{aligned}
$$

Proof. Since $a(n-k) \leq a(n)$ for $k \leq n$ the left hand side in (13) is bounded by

$$
\sum_{n=1}^{N} \sum_{m=n}^{N} \frac{\bar{a}(n) a(n)}{n m a(n) a(m)}=\sum_{n=1}^{N} \sum_{m=n}^{N} \frac{\left(1+\beta_{n}\right) a(n)}{n m a(m)} .
$$

By Lemma 2 it follows that

$$
\sum_{n=1}^{N} \sum_{m=n}^{N} \frac{\left(1+\beta_{n}\right) a(n)}{n m a(m)} \leq\left(1+\sup _{n \geq 1} \beta_{n}\right)\left(\varepsilon+\Phi(N, \varepsilon)+(\log N)^{-1}\right)(1+\log N) \log N
$$

Lemma 4. For any $\varepsilon>0$ and $N \geq 2$

$$
2 \sum_{n=1}^{N} \frac{1}{n a(n)} \sum_{m=n+1}^{N} \frac{1}{m a(m)} \sum_{k=0}^{n} l_{k} a(m-k) \leq(1+\log N)^{2}+2 \sum_{n=1}^{N} \frac{\beta_{n}}{n} \log N .
$$


Proof. Write

$$
\begin{aligned}
2 \sum_{n=1}^{N} & \sum_{m=n+1}^{N} \frac{1}{n m a(n) a(m)} \sum_{k=0}^{n} a(m-k) l_{k} \\
& \leq 2 \sum_{n=1}^{N} \sum_{m=n+1}^{N} \frac{1+\beta_{n}}{n m}=(1+\log N)^{2}+2 \sum_{n=1}^{N} \frac{\beta_{n}}{n} \log N
\end{aligned}
$$

Lemma 5. For any $\varepsilon>0$ and $N \geq 2$

$$
\sum_{k=1}^{N} \frac{\beta_{k}}{k} \leq\left(\gamma(1) \varepsilon+\gamma\left(N^{\varepsilon}\right)\right)(1+\log N),
$$

where

$$
\gamma(N)=\sup \left\{\beta_{k}: N \leq k\right\}
$$

Proof. Obvious.

Proposition 3. For every $\varepsilon>0$ and $N \geq 2$

$\operatorname{Var}\left(\frac{1}{\log N} T_{N}\right) \leq\left[2 \gamma\left(N^{\varepsilon}\right)+(5+4 \gamma(1))\left(\varepsilon+\Phi(N, \varepsilon)+(\log N)^{-1}\right)\right]\left(\frac{1+\log N}{\log N}\right)$.

Proof. Since $E\left(T_{N}\right)=\log N$ the statement follows immediately from Lemmas $1,3,4$, and 5 .

Proof of Theorem 1. By Proposition 3 and Chebychev's inequality we obtain that

$$
\frac{1}{\log N} \sum_{n=1}^{N} \frac{1}{n a(n)} S_{n} \rightarrow 1
$$

in probability with respect to $m$ restricted to the set $A$.

The theorem then follows from general arguments. The convergence of (14) in probability on $A$ is equivalent to the fact that every subsequence contains a further subsequence $N_{j}$ so that (14) converges along this subsequence a.s. on $A$. But the set of convergence is $T$-invariant as is the limit, and so by ergodicity this convergence is to 1 a.s. with respect to $m$, and hence the convergence in (14) is in measure on any set of finite measure. In order to obtain the statement for arbitrary $f \in L_{1}(m)$ apply Hopf's Ergodic Theorem to every a.s. convergent subsequence.

Proof of Theorem 2. The proof is similar to that one of Theorem 1.

Under the assumptions of the theorem

$$
E\left(T_{N}-E\left(T_{N}\right)\right)^{2}=O\left((\log N)^{2-\delta}\right),
$$

where $\delta=\min (\varepsilon, \gamma)$. This follows from Proposition 3. Choose $r$ so large that $r \delta>1$. By the Borel-Cantelli Lemma it follows that

$$
\lim _{p \rightarrow \infty} \frac{1}{p^{r} \log 2} T_{2^{p^{r}}}=1
$$

almost surely. The theorem then follows from the monotonicity of $T_{N}$ and the asymptotic equivalence $\lim _{p \rightarrow \infty} p^{r}(p+1)^{-r}=1$. 
Proof of Corollary 1. If $T$ is a Markov shift, then we may take $A=\left\{\left(x_{i}\right)_{i \geq 1}\right.$ : $\left.x_{1}=a\right\}$ where $a$ denotes some fixed state. It is well known that the corresponding $\beta_{n}=0$, where $\beta_{n}$ is defined by (10).

\section{CHUNG-ERdöS AVERAGES FOR INNER FUNCTIONS}

Let $T:\left(\mathbb{R}^{2}\right)^{+} \rightarrow\left(\mathbb{R}^{2}\right)^{+}$be an analytic endomorphism of the upper halfplane $\left(\mathbb{R}^{2}\right)^{+}$. It is well known that $T$ has a representation

$$
T(z)=\alpha z+\beta+\int_{\boldsymbol{R}} \frac{1+t z}{t-z} \mu(d t),
$$

where $\alpha \geq 0, \beta \in \mathbb{R}$, and $\mu$ denotes some positive measure on the real line. $T$ is called an inner function if for a.e. $x \in \mathbb{R}$

$$
\lim _{y \rightarrow 0} T(x+i y)=: T(x) \in \mathbb{R},
$$

i.e., $\mu$ is singular with respect to the one-dimensional Lebesgue measure. An inner function is called odd if $T(-x)=-T(x)(x \in \mathbb{R})$, or equivalently: $\beta=0$ and $\mu$ is symmetric, i.e., $\mu(A)=\mu(-A)$ for $A \subset \mathbb{R}$, see [1, §2]. In this section we shall assume that $T$ is odd and we shall consider the dynamical system defined by $T$ on the real line $\mathbb{R}$. Moreover, we shall assume that $\alpha=1$, since in this case the Lebesgue measure $\lambda$ on $\mathbb{R}$ is an invariant measure (see [9]).

Each point $z=a+i b \in\left(\mathbb{R}^{2}\right)^{+}$defines a Cauchy density function

$$
\phi_{z}(t)=\frac{b}{\pi\left[(x-a)^{2}+b^{2}\right]} .
$$

Define

$$
a(k)=\sum_{j=1}^{k} u_{j},
$$

where

$$
u_{j}=\int_{\boldsymbol{R}} \phi_{i} \circ T^{j} \cdot \phi_{i} d \lambda .
$$

In the subsequent proof we shall make use of the following fact about the relation of $T$ and $\phi_{z}$.

Lemma 6 [9]. Denote by $P_{z}$ the Cauchy distribution with density $\phi_{z}$. Then

$$
P_{z} \circ T^{-1}=P_{T(z)}
$$

and

$$
\widehat{T} \phi_{z}=\phi_{T(z)} .
$$

Using Lemma 6, it is shown in Corollary 3.5 in [1] that $T$ is conservative if and only if $a(n) \rightarrow \infty$ as $n \rightarrow \infty$ and in this case, $T$ is pointwise dual ergodic with return sequence $a(n)$. The main result in this section is 
Theorem 3. Let $T$ be a conservative odd inner function of the upper half-plane restricted to the real line. Then

$$
\lim _{N \rightarrow \infty} \frac{1}{\log a(N)} \sum_{k=1}^{N} \frac{1}{a(k)} \phi_{i} \circ T^{k}=1
$$

and for every function $f \in L_{1}^{+}(\lambda)$,

$$
\lim _{N \rightarrow \infty} \frac{1}{\log a(N)} \sum_{k=1}^{N} \frac{1}{a(k)} f \circ T^{k}=\int_{\boldsymbol{R}} f d \lambda
$$

almost surely.

Proof. It suffices to prove the statement (21), as (22) follows from (21), by Proposition 2 and Hopf's Ergodic Theorem.

Next observe that it suffices to show (21) $P_{i}$-almost surely, since $\lambda$ and the Cauchy distribution $P_{i}$ are equivalent.

We denote by $E$ the expectation with respect to the measure $P_{i}$ and by

$$
T_{N}=\sum_{n=1}^{N} \frac{1}{a(n)} \phi_{i} \circ T^{n} .
$$

Then

$$
\begin{aligned}
E\left(T_{N}^{2}\right)= & \sum_{n=1}^{N} \frac{1}{a(n)^{2}} \int \phi_{i}^{2} \circ T^{n} \cdot \phi_{i} d \lambda \\
& +2 \sum_{n=1}^{N-1} \sum_{m=n+1}^{N} \frac{1}{a(n) a(m)} \int \phi_{i} \circ T^{n} \cdot \phi_{i} \circ T^{m} \cdot \phi_{i} d \lambda
\end{aligned}
$$

and

$$
\left(E\left(T_{N}\right)\right)^{2}=\sum_{n=1}^{N} \frac{u_{n}^{2}}{a(n)^{2}}+\sum_{n=1}^{N-1} \sum_{m=n+1}^{N} \frac{u_{n} u_{m}}{a(n) a(m)} .
$$

Note that $T\left(i \mathbb{R}_{+}\right) \subset i \mathbb{R}_{+}$. Therefore we can define $c_{n}$ by $T^{n}(i)=c_{n} i$. It is not hard to see that $c_{n} \uparrow \infty$ and indeed that $c_{n}>>\sqrt{n}$ (see [1]). Then by (19) and $(20)$

$$
\begin{aligned}
\int \phi_{i} \cdot \phi_{i} \circ T^{n} \cdot \phi_{i} \circ T^{n+k} d \lambda & =\int \phi_{T^{n}(i)} \cdot \phi_{i} \cdot \phi_{i} \circ T^{k} d \lambda \\
& =\int \widehat{T}^{k}\left(\phi_{i} \cdot \phi_{c_{n} i}\right) \cdot \phi_{i} d \lambda .
\end{aligned}
$$

For any $b \in \mathbb{R}_{+}$we have

$$
\begin{aligned}
\pi^{2} \phi_{i} \cdot \phi_{b i}(x) & =\frac{b}{\left[x^{2}+1\right]\left[x^{2}+b^{2}\right]}=\frac{b}{\left[x^{2}+1\right]\left[b^{2}-1\right]}-\frac{b}{\left[x^{2}+b^{2}\right]\left[b^{2}-1\right]} \\
& =\frac{b \pi}{b^{2}-1} \phi_{i}-\frac{\pi}{b^{2}-1} \phi_{b i}
\end{aligned}
$$

and

$$
\pi^{2} \int \phi_{i} \cdot \phi_{b i} d \lambda=\frac{\pi b}{b^{2}-1}-\frac{\pi}{b^{2}-1}=\frac{\pi}{b+1}
$$


By (20)

$$
\begin{aligned}
\widehat{T}^{k}\left(\phi_{i} \cdot \phi_{c_{n} i}\right) & =\widehat{T}^{k}\left(\frac{c_{n}}{\pi\left(c_{n}^{2}-1\right)} \phi_{i}-\frac{1}{\pi\left(c_{n}^{2}-1\right)} \phi_{c_{n} i}\right) \\
& =\frac{c_{n}}{\pi\left(c_{n}^{2}-1\right)} \phi_{c_{k} i}-\frac{1}{\pi\left(c_{n}^{2}-1\right)} \phi_{c_{n+k} i}
\end{aligned}
$$

and integrating with respect to $P_{i}$ yields (use (23), (24), and (25))

$$
\begin{aligned}
\int \phi_{i} \cdot \phi_{i} \circ T^{n} \cdot \phi_{i} \circ T^{n+k} d \lambda & =\int \widehat{T}^{k}\left(\phi_{i} \cdot \phi_{c_{n} i}\right) \cdot \phi_{i} d \lambda \\
& =\frac{1}{\pi\left(c_{n}^{2}-1\right)}\left[c_{n} \int \phi_{i} \cdot \phi_{c_{k} i} d \lambda-\int \phi_{i} \cdot \phi_{c_{n+k} i} d \lambda\right] \\
& =\frac{1}{\pi^{2}\left(c_{n}^{2}-1\right)}\left[\frac{c_{n}}{c_{k}+1}-\frac{1}{c_{n+k}+1}\right] .
\end{aligned}
$$

It follows from this and (24) that

$$
\begin{aligned}
E\left(T_{N}^{2}\right) & -\left(E\left(T_{N}\right)\right)^{2} \\
= & \sum_{n=1}^{N} \frac{1}{a(n)^{2}}\left[\int \phi_{i}^{2} \cdot \phi_{c_{n} i} d \lambda-\frac{1}{\pi^{2}\left(c_{n}+1\right)^{2}}\right]+2 \sum_{n=1}^{N-1} \frac{1}{\pi^{2} a(n)} \mathscr{D}(N, n)
\end{aligned}
$$

where

$$
\begin{aligned}
\mathscr{D}(N, n)= & \sum_{k=1}^{N-n} \frac{1}{a(n+k)\left(c_{n}^{2}-1\right)}\left(\frac{c_{n}}{c_{k}+1}-\frac{1}{c_{n+k}+1}\right) \\
& -\sum_{k=n+1}^{N} \frac{1}{a(k)\left(c_{n}+1\right)\left(c_{k}+1\right)} .
\end{aligned}
$$

Now

$$
\sum_{n=1}^{N} \frac{1}{a(n)^{2}} \int \phi_{i}^{2} \cdot \phi_{c_{n} i} d \lambda \leq \sum_{n=1}^{N} \frac{u_{n}}{\pi a(n)^{2}}=O(\log a(N))
$$

and

$$
\begin{aligned}
\sum_{k=n+1}^{N-n} & \frac{1}{a(n+k)\left(c_{n}^{2}-1\right)}\left(\frac{c_{n}}{c_{k}+1}-\frac{1}{c_{n+k}+1}\right)-\frac{1}{a(k)\left(c_{n}+1\right)\left(c_{k}+1\right)} \\
\leq & \sum_{k=n+1}^{N-n} \frac{1}{a(k)\left(c_{k}+1\right)}\left(\frac{c_{n}-1}{c_{n}^{2}-1}-\frac{1}{c_{n}+1}\right) \\
& +\sum_{k=n+1}^{N-n} \frac{1}{a(n+k)\left(c_{n}^{2}-1\right)}\left(\frac{1}{c_{k}+1}-\frac{1}{c_{n+k}+1}\right) \\
= & O\left(\sum_{k=n+1}^{N-n} \frac{1}{a(k)\left(c_{n}+1\right)^{2}\left(c_{k}+1\right)}\right) \\
= & O\left(\sum_{k=n+1}^{N-n} \frac{u_{n}^{2} u_{k}}{a(k)}\right)=O\left(u_{n}^{2} \log a(N)\right) .
\end{aligned}
$$


Moreover

$$
\begin{aligned}
\sum_{k=1}^{n} \frac{1}{a(n+k)\left(c_{n}^{2}-1\right)}\left[\frac{c_{n}}{c_{k}+1}-\frac{1}{c_{n+k}+1}\right] & =O\left(\sum_{k=1}^{n} \frac{u_{n} u_{k}}{a(n+k)}\right) \\
& =O\left(\frac{u_{n}}{a(n)} \sum_{k=1}^{n} u_{k}\right)=O\left(u_{n}\right) .
\end{aligned}
$$

Using these estimates in (26) we arrive at

$$
E\left(T_{N}\right)^{2}-\left(E\left(T_{N}\right)\right)^{2}=O\left(\left(1+\sum_{n=1}^{N-1} \frac{u_{n}^{2}}{a(n)}\right) \log a(N)\right)=O(\log a(N)),
$$

since $\sum_{n \geq 1} u_{n}^{2}(a(n))^{-1}<\infty$. This is because $c_{n} \geq d \sqrt{n}$ for some constant $d>0$, and $u_{n} \downarrow$, hence $u_{n} \leq n^{-1} a(n)$ and

$$
\sum_{k=1}^{\infty} \frac{u_{k}^{2}}{a(k)} \leq \frac{1}{d} \sum_{k=1}^{\infty} k^{-3 / 2}<\infty
$$

It follows that

$$
\operatorname{Var}\left(\sum_{k=1}^{N} \frac{1}{a(k)} \phi_{i} \circ T^{k}\right)=O(\log a(N)) .
$$

Choose a sequence $N_{k}$ such that $\sum_{k \geq 1}\left(\log a\left(N_{k}\right)\right)^{-1}<\infty$ and

$$
\frac{\log a\left(N_{k}\right)}{\log a\left(N_{k+1}\right)} \rightarrow 1
$$

e.g., choose $N_{k}=\inf \left\{m: a(m)>\exp \left(k^{2}\right)\right\}$. Then, by the Borel-Cantelli Lemma, for any $\eta>0$,

$$
\sum_{k=1}^{\infty} P_{i}\left(\left\{x: T_{N_{k}}-E\left(T_{N_{k}}\right) \geq \eta \log a\left(N_{k}\right)\right\}\right)=O\left(\eta^{-2} \sum_{k=1}^{\infty} \frac{1}{\log a\left(N_{k}\right)}\right)<\infty .
$$

Hence $\lim _{k \rightarrow \infty} T_{N_{k}} / \log a\left(N_{k}\right)=1$ a.s. and therefore we obtain

$$
\lim _{N \rightarrow \infty} \frac{T_{N}}{\log a(N)}=1 \quad \text { a.s. }
$$

as in the proof of Theorem 2 .

Remark 3. If $a(n)$ is regularly varying with index $>0$, then we also have that

$$
\lim _{N \rightarrow \infty} \frac{1}{\log N} \sum_{n=1}^{N} \frac{1}{n a(n)} S_{n} f=\int_{R} f d \lambda \quad \text { a.s. }
$$

by Proposition 1 .

We show finally that there exist odd inner functions with $a(n)$ any regularly varying sequence of index in $(0,1 / 2)$. This result extends Lemma 3.7 in [1].

Proposition 4. Suppose $0<\gamma<1 / 2$ and that $b(n)$ is regularly varying with index $\gamma$ as $n \rightarrow \infty$. Then there is an odd inner function

$$
T(x)=x+\int_{\boldsymbol{R}} \frac{1+t x}{t-x} d \mu(t)
$$

such that $a(n) \sim b(n)$ as $n \rightarrow \infty$. 
Proof. Let

$$
T(x)=x+\int_{\boldsymbol{R}} \frac{1+t x}{t-x} d \mu(t)
$$

be an odd inner function where the symmetric measure $\mu$ has a tail distribution

$$
c_{\mu}(b)=\mu(\{t:|t| \geq b\}),
$$

which is regularly varying at infinity with index $-\alpha$, where $\alpha \in(1,2)$. Let $T^{n}(i)=b_{n} i$. By (15), the definition of $T$, we obtain that

$$
b_{n+1}=b_{n}\left(1+F\left(b_{n}\right)\right) \quad(n \geq 1),
$$

where

$$
F(b)=\int_{-\infty}^{\infty} \frac{t^{2}+1}{t^{2}+b^{2}} \mu(d t) .
$$

It can be shown as in the proof of Lemma 3.7 in [1] that

$$
F(b)=b^{-2}+2\left(1-b^{-2}\right) \int_{0}^{\infty} c_{\mu}(b z) \frac{z}{\left(z^{2}+1\right)^{2}} d z \sim d_{\alpha} c_{\mu}(b),
$$

where

$$
0<d_{\alpha}=2 \int_{0}^{\infty} \frac{z^{1-\alpha}}{\left(z^{2}+1\right)^{2}} d z<\infty .
$$

Hence $F(b)$ is also regularly varying at infinity with index $-\alpha$.

Consequently $C(b)=(F(b))^{-1}$ is regularly varying with index $\alpha$ and and satisfies

$$
C\left(b_{n+1}\right)-C\left(b_{n}\right) \rightarrow \alpha \quad \text { as } n \rightarrow \infty .
$$

Therefore $C\left(b_{n}\right) \sim n \alpha$ and

$$
b_{n} \sim C^{-1}(n \alpha) \sim \alpha^{1 / \alpha} C^{-1}(n)
$$

as $n \rightarrow \infty$, and is $\alpha^{-1}$-regularly varying.

Since $1<\alpha<2, \sum_{n>1} b_{n}^{-1}=\infty$, whence $T$ is conservative and the return sequence satisfies (by (17), (18), and (24))

$$
a(n) \sim \frac{1}{\pi} \sum_{k=1}^{n} \frac{1}{b_{k}} \sim \frac{n}{b_{n} \pi\left(1-\alpha^{-1}\right)} \sim \frac{1}{\pi\left(1-\alpha^{-1}\right) \alpha^{1 / \alpha}} \frac{n}{C^{-1}(n)} .
$$

In order to obtain the proposition from the last statement, let $0<\gamma<1 / 2$ and a $\gamma$-regularly varying sequence $b(n)$ be given. Define $\alpha=\frac{1}{1-\gamma} \in(1,2)$, and set

$$
\psi(x)=\frac{1}{\pi\left(1-\alpha^{-1}\right) \alpha^{1 / \alpha}} \frac{x}{b(x)} .
$$

Let $\mu$ be a symmetric measure on $\mathbb{R}$, singular with respect to Lebesgue measure, such that

$$
c_{\mu}(b) \sim \frac{1}{d_{\alpha} \psi^{-1}(b)}
$$

as $b \rightarrow \infty$. From the above, it follows that if

$$
T(x)=x+\int_{\boldsymbol{R}} \frac{1+t x}{t-x} d \mu(t)
$$

then the associated sequence $a(n)$ defined by (17) satisfies $a(n) \sim b(n)$. 


\section{REFERENCES}

1. J. Aaronson, Ergodic theory for inner functions of the upper half plane, Ann. Inst. H. Poincaré. Anal. Non Linéaire 14 (1978), 233-253.

2. __ The asymptotic distributional behaviour of transformations preserving infinite measures, J. D'Analyse Math. 39 (1981), 203-234.

3. __ Random f-expansions, Ann. Prob. 14 (1986), 1037-1057.

4. T. Bedford and A. M. Fisher, Analogues of the Lebesgue density theorem for fractal subsets of the reals and integers, Proc. London Math. Soc. (to appear).

5. K. L. Chung and P. Erdös, Probability limit theorems assuming only the first moment. I, Mem. Amer. Math. Soc. 6 (1951).

6. W. Feller, An Introduction to probability theory and its applications 2, Wiley, New York, 1971.

7. A. M. Fisher, A pathwise central limit theorem for random walks, Ann. Prob. (to appear).

8. Integer Cantor sets and an order-two ergodic theorem, J. D'Analyse Math. (to appear).

9. G. Letac, Which functions preserve Cauchy laws, Proc. Amer. Math. Soc. 67 (1977), 277286.

10. M. Thaler, Transformations on $[0,1]$ with infinite invariant measures, Israel J. Math. 46 (1983), 67-96.

School of Mathematical Sciences, Tel Aviv University, Ramat Aviv, 69978 Tel Aviv, ISRAEL

InSTitut Für Mathematische Stochastik, Universität Göttingen, Lotzestr.13, 3400 GöTtINGEN, GeRMANY

Department of Mathematical Sciences, Memphis State University, Memphis, TENNESSEE, 38152 